\title{
LES study of microphysical variability bias in shallow cumulus
}

\author{
Yefim Kogan \\ NorthWest Research Associates, Redmond, WA, USA \\ Correspondence to: Yefim Kogan (ykogan@nwra.com)
}

Received: 9 January 2017 - Revised: 29 March 2017 - Accepted: 3 April 2017 - Published: 2 May 2017

\begin{abstract}
Subgrid-scale (SGS) variability of cloud microphysical variables over the mesoscale numerical weather prediction (NWP) model has been evaluated by means of joint probability distribution functions (JPDFs). The latter were obtained using dynamically balanced Large Eddy Simulation (LES) model dataset from a case of marine trade cumulus initialized with soundings from Rain in Cumulus Over the Ocean (RICO) field project. Bias in autoconversion and accretion rates from different formulations of the JPDFs was analyzed. Approximating the 2-D PDF using a "generic" (fixed-in-time), but variable-in-height JPDFs give an acceptable level of accuracy, whereas neglecting the SGS variability altogether results in a substantial underestimate of the grid-mean total conversion rate and producing negative bias in rain water. Nevertheless the total effect on rain formation may be uncertain in the long run due to the fact that the negative bias in rain water may be counterbalanced by the positive bias in cloud water. Consequently, the overall effect of SGS neglect needs to be investigated in direct simulations with a NWP model.
\end{abstract}

\section{Introduction}

Formulation of microphysical processes in meso and large scale models requires accounting for subgrid-scale (SGS) variability; its neglect can lead to substantial bias in calculations of microphysical process rates (Pincus and Klein, 2000; Larson et al., 2001, 2012; Kogan and Mechem, 2014, KM14 hereafter, Nelson et al., 2016). SGS microphysical variability can be represented using probability distribution functions (PDFs). The greatest challenge in this approach is linking the PDF parameters to the grid-mean prognostic variables (so called "closure" relationships). KM14 present a detailed review of the various recent PDF approaches, the most sophisticated of which employs joint analytic PDFs of liquid water potential temperature, total water, and vertical velocity (Cloud Layers Unified by Binomials [CLUBB], Golaz et al., 2002; Larson and Golaz, 2005). The CLUBB approach uses theoretical considerations and a number of a-priori assumptions about the shape of the distributions, obtained from LES output and aircraft observations.

KM14 use a series of Large Eddy Simulations (LES) of trade wind shallow cumulus based on the RICO field campaign (Rauber et al., 2007). These clouds are one of the most prevalent cloud types on Earth and play an important role in establishing the thermodynamic structure of the lower atmosphere in the trade latitudes. In KM14 the absolute errors of various PDF formulations were evaluated; the goal of this paper is to evaluate the errors in more detail and analyze how they may affect precipitation formation at different stages of cloud and rain formation

\section{Model and dataset}

The model employed in our simulations is a version of the Cooperative Institute for Mesoscale Meteorological Studies (CIMMS) LES (Kogan et al., 1995; Khairoutdinov and Kogan, 1999) called SAMBM (System for Atmospheric Modeling - Bulk Microphysics) (Khairoutdinov and Kogan, 2000; Kogan, 2013). This new parameterization has been tested against simulations using the CIMMS LES with explicit microphysics (SAMEX, Kogan et al., 2012) in case studies of northeast Atlantic marine stratocumulus (the Atlantic Stratocumulus Experiment [ASTEX], Albrecht et al., 1995) and marine trade cumulus based on the Rain in Cumulus over the Ocean (RICO) field campaign (vanZanten et al., 2011). 
The simulations were performed in the integration domain $25.6 \times 25.6 \mathrm{~km}^{2}$ using horizontal resolution of $100 \mathrm{~m}$ and vertical resolution of $40 \mathrm{~m}$. Our integration domain size roughly correspond to the grid size of a NWP model. The analysis dataset is selected from clouds evolving during the second half of the $24 \mathrm{~h}$ simulation when deeper clouds appear and precipitation is most intense.

The KM14 methodology includes two distinct elements for deriving the PDF parameterization. The first is the use of a layered approach in formulating the PDF under which we subdivide the $4 \mathrm{~km}$ model vertical domain into ten, 400$\mathrm{m}$-thick layers. The resulting increase of the data volume in each layer leads to the increase of statistical confidence of the PDF calculations. The second element is the formulation of the PDFs in non-dimensional space by normalizing the microphysical variables by their layer-mean values.

\section{Results}

We consider two joint probability distribution functions (JPDFs) which enter the expressions for microphysical autoconversion and accretion rates. The first JPDF is used for calculation of autoconversion; it depends on cloud-drop number concentration, $N_{\mathrm{c}}$ and cloud water mixing ratio, $q_{\mathrm{c}}$. The second one is needed for calculation of the accretion; it depends on $q_{\mathrm{c}}$ and rain water mixing ratio, $q_{\mathrm{r}}$. Accounting for cloud variability (as described by PDFs) allows calculating unbiased microphysical process rates over a mesoscale NWP model grid. While the JPDFs at each mesoscale model grid point, in general, depend on the grid averages of resolvable prognostic variables, formulation of such dependencies (closure schemes) is a very complex and formidable task; a more modest and realistic approach is to use simplified approximations of JPDFs. Our goal here is evaluation of the errors introduced by such approximations in calculations of microphysical processes of autoconversion and accretion and their sum total: "conversion" rates.

As was shown in KM14, the JPDFs vary in the vertical and depend on parameters characterizing cloud system environment; the latter change as cloud system evolves with time during the simulation. Figure 1 shows that domain averaged TKE and LWP increased by $50 \%$ over the course of $12 \mathrm{~h}$, while rain water path (RWP) increased four-fold. Both LWP and RWP vary significantly during the course of simulation. We archive hourly datasets and derived time dependent JPDFs from these datasets. The JPDF variation in time, therefore, corresponds to changing environmental conditions.

The fine resolution of the LES model allows us to calculate the "exact" conversion rates which serve as a benchmark for evaluating different approximations. Based on the values of $q_{\mathrm{c}}, N_{\mathrm{c}}$, and $q_{\mathrm{r}}$ at each LES grid point, we calculate the autoconversion and accretion rates, which are then averaged for each layer to provide "exact" conversion rates at this layer.

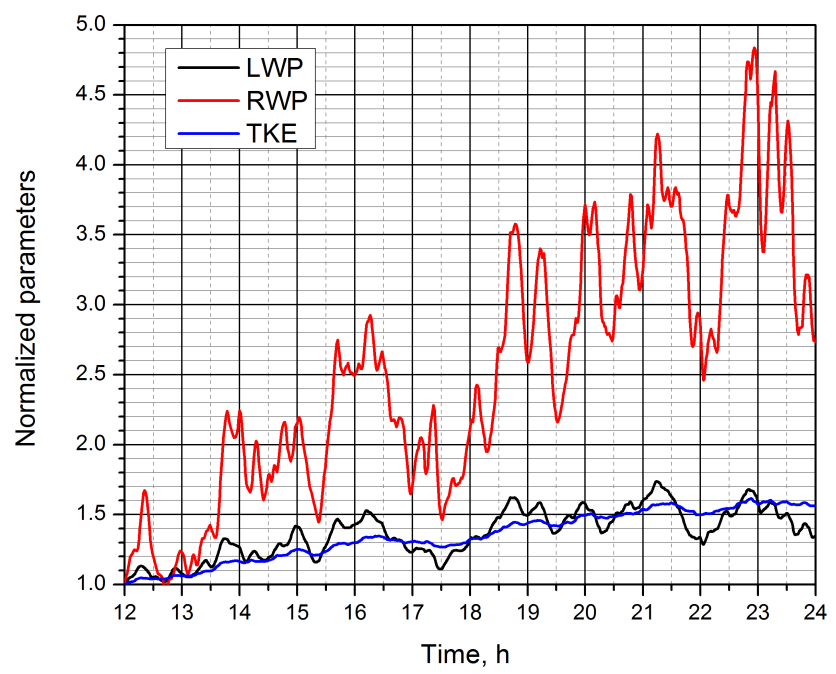

Figure 1. The evolution of domain averaged liquid water path (LWP), rain water path (RWP), and turbulent kinetic energy (TKE) during the second half of the simulation. All parameters are normalized by their corresponding value at $12 \mathrm{~h}$.

In addition to the time dependent JPDFs, we consider the case when the time dependence (and hence varying environmental conditions) is neglected. These JPDFs are calculated using the entire data set from the whole simulation; they may be referred to as a "generic" shallow cumulus JPDFs. For comparison we also consider an approximation when the JPDFs are neglected altogether, and the conversion rates are calculated using only the layer-mean variables. This case serves as the measure of improvement when SGS variability is included.

The errors of different JPDF approximations are defined as: $\varepsilon=(A / E-1) 100$, where $A$ and $E$ are the approximated and the corresponding "exact" conversion rates. Figure 2 shows both the cumulative and density distribution of the errors for autoconversion and accretion rates. The smallest errors are produced by JPDFs which vary both in time and in the vertical. Although the time dependent JPDFs are tuned each hour to the clouds they represent, nevertheless, the conversion rates still show errors (Fig. 2, black curve). This is explained by the use of rather coarse resolution in discretizing the JPDFs (only $15 \times 15$ bins and limiting the variability of dimensionless variables to a range from 0 to 3 ).

Remarkably, the errors coming from JPDF which are derived from the entire $12 \mathrm{~h}$ dataset and, therefore, neglect timedependence of JPDF, are not significantly larger than the errors produced by using the time-dependent JPDFs (compare black and red curves in Fig. 2). These simplified fixed in time "generic" JPDFs can be implemented in the NWP models as a proxy for trade wind shallow cumulus clouds.

Neglecting SGS altogether introduces significant negative bias for autoconversion rates with errors in the -60 to $-80 \%$ range. The SGS bias in the accretion rates is approximately 

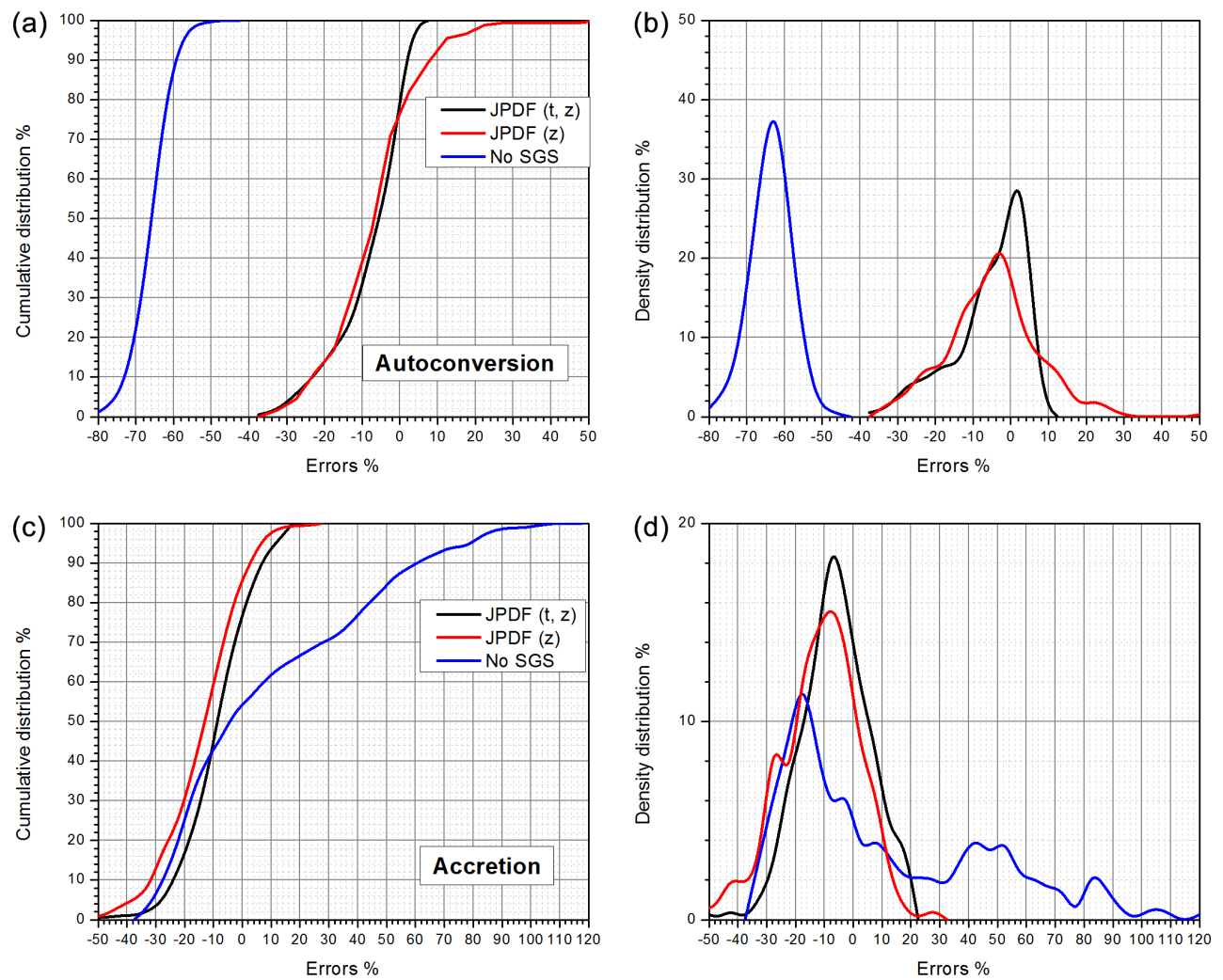

Figure 2. The cumulative (a, c) and density (b, d) distributions of errors of different approximations for JPDFs in calculations of autoconversion (a) and accretion (c) rates. The black curve represents JPDF varying in time and in the vertical. The red curve represents case with the "generic" (independent of time), but height dependent JPDF. The blue curve refers to a case with SGS variability neglected.
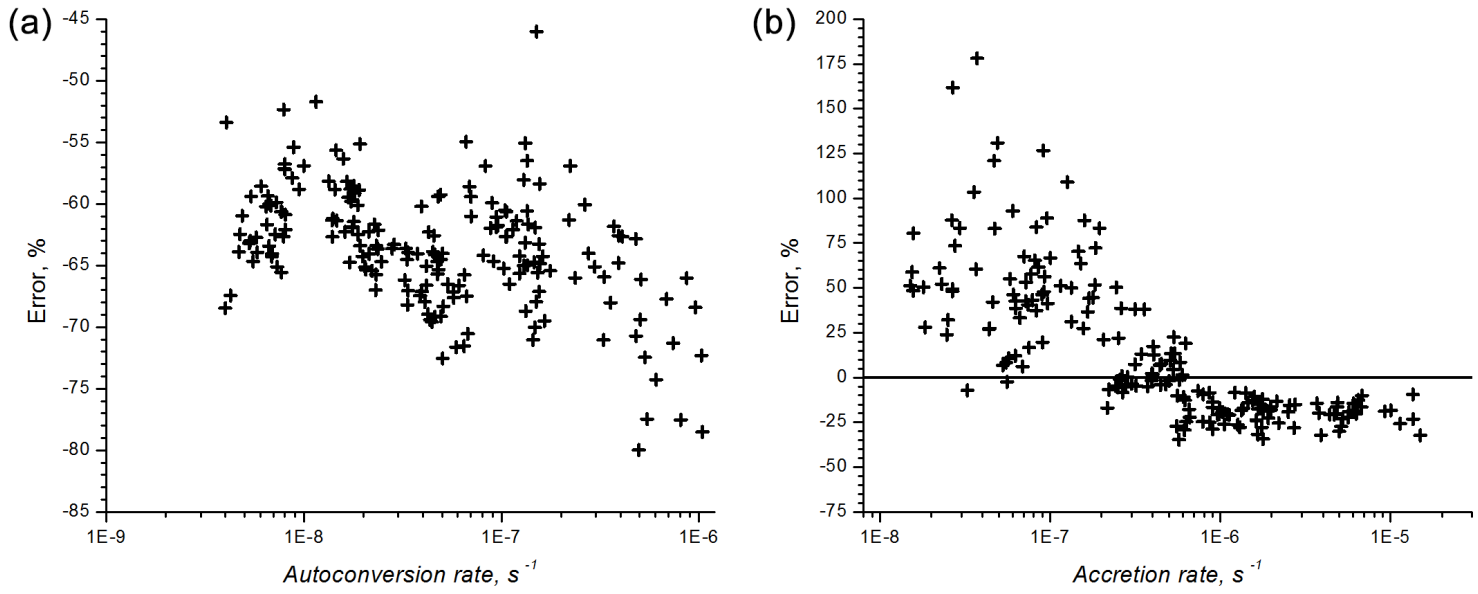

Figure 3. The error dependence on the rate value. (a) - autoconversion, (b) - accretion rate.

equally divided between negative and positive errors. The peak of negative errors is at $-30 \%$ and they do not exceed more than $-40 \%$. The positive errors lie in a wider range and have a tail extending up to $+120 \%$.

Important information about the effect of the errors on rain production can be gained when considering their dependence on the value of the rate itself (Fig. 3). For autoconversion, the errors increase with the increase of the value of the rate, e.g., the small autoconversion rates have the smallest errors (in the $-65-50 \%$ range), while the largest autoconversion rates have also the largest negative bias (up to $-80 \%$ ). For accretion, the small to moderate accretion rates are positively biased (up to $+120 \%$ ), while the moderate to strong accretion rate have consistently negative bias (on the order of -25 

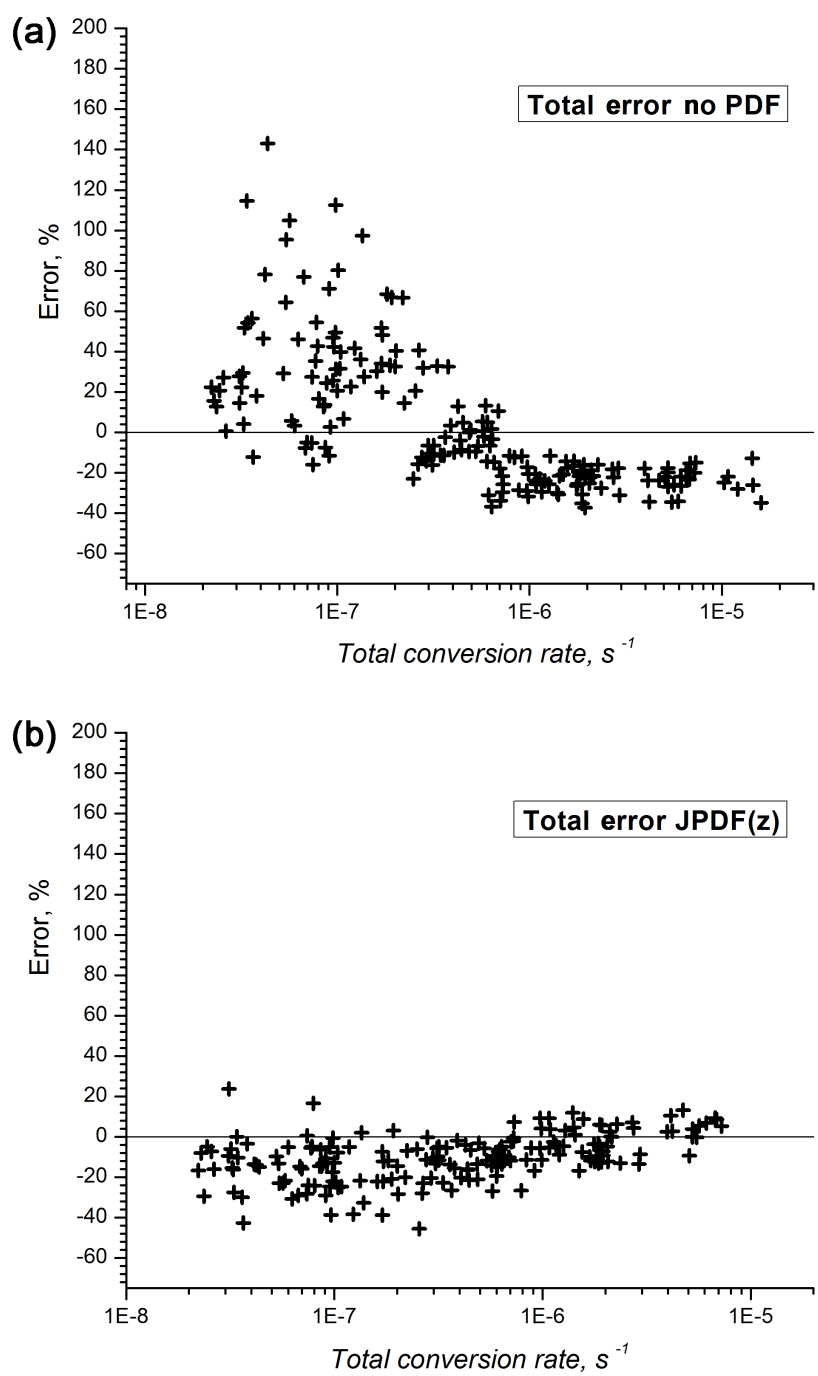

Figure 4. The total conversion rate error as a function of the rate. Panel (a) for case with SGS neglected; (b) for the total "generic" conversion rate.

to $-40 \%)$. In the model equations both autoconversion and accretion rates are acting simultaneously, so it is useful to analyze the effect of the sum of these rates - the total conversion rate shown in Fig. 4. When SGS is neglected, the bias of the total rate essentially follows the bias of the accretion rate; the latter is generally larger than autoconversion, especially when clouds are at mature stage and precipitation is already formed. Specifically, the bias is negative for rates larger than $0.7 \times 10^{-7} \mathrm{~s}^{-1}$.

The negative bias is eliminated when JPDFs are employed. The bottom panel in Fig. 4 shows the bias for the total conversion rates using "generic" JPDF. For large values of the rate, the overall bias is decreased and varies in the range of -20 to $+10 \%$. For small to moderate rates the bias is mostly confined to a narrow negative range of 0 to $-30 \%$.
While results in Fig. 4a may suggest that the bias for the total conversion rate is mostly negative for the larger values of the rate, nevertheless it does not warrant a conclusion that neglecting the SGS variability will result in underestimation of rain formation. This is because one needs to consider the fact that when total (underestimated) conversion rate is being added to the rain water mixing ratio $q_{\mathrm{r}}$, it is simultaneously subtracted from the cloud water mixing ratio $q_{\mathrm{c}}$. As a result, the positive bias in $q_{\mathrm{c}}$ may counterbalance the negative bias in $q_{\mathrm{r}}$. Consequently the overall effect of SGS neglect may be uncertain and needs to be investigated in direct simulations using NWP models.

\section{Conclusions}

Previously in KM14 we evaluated the absolute total errors on various PDF formulations. In this study we evaluate the errors in more detail and analyze how they may affect precipitation formation at different stages of cloud and rain formation. It might be expected that neglecting SGS variability may lead to slow rain initiation because of the significant underestimation of autoconversion rates, however, this effect may be somewhat compensated by the overestimation of accretion rates at the beginning. At the later stages of rain development both autoconversion and accretion rate are underestimated, and, thus, lead to smaller values of the rain water mixing ratio. However, the underestimated values of the conversion rates which are subtracted from the cloud water mixing ratio, will result in its overestimation, therefore, counterbalancing the negative bias in $q_{\mathrm{r}}$. More definite effect of SGS variability neglect can only be evaluated by sensitivity studies using different PDF formulations in the NWP model.

Data availability. Sounding data for simulations of RICO trade wind convective clouds is available at doi:10.1029/2011MS000056 (vanZanten et al., 2011).

Competing interests. The authors declare that they have no conflict of interest.

Acknowledgements. This investigation was supported by the ONR grant N00014-16-1-2487. The computing for this project was performed at the University of Oklahoma Supercomputing Center for Education and Research (OSCER). The constructive comments of the anonymous reviewer are appreciated.

Edited by: D. Reinert

Reviewed by: two anonymous referees 


\section{References}

Albrecht, B. A., Bretherton, C. S., Johnson, D. W., Schubert, W. H., and Frisch, A. S.: The Atlantic stratocumulus transition experiment - ASTEX, B. Am. Meteorol. Soc. 76, 889-904, 1995.

Golaz, J.-C., Larson, V. E., and Cotton, W. R.: A PDF-based model for boundary layer clouds. Part I: Method and model description, J. Atmos. Sci., 59, 3540-3551, 2002.

Khairoutdinov, M. F. and Kogan, Y. L.: A large eddy simulation model with explicit microphysics: Validation against aircraft observations of a stratocumulus-topped boundary layer, J. Atmos. Sci., 56, 2115-2131, 1999.

Khairoutdinov, M. and Kogan, Y. L.: A new cloud physics parameterization for large-eddy simulation models of marine stratocumulus, Mon. Weather Rev., 128, 229-243, 2000.

Kogan, Y. L.: A Cumulus Cloud Microphysics Parameterization for Cloud-Resolving Models, J. Atmos. Sci., 70, 1423-1436, 2013.

Kogan, Y. L. and Mechem, D. B.: A PDF based microphysics parameterization for shallow cumulus cloud, J. Atmos. Sci., 71, 1070-1089, 2014.

Kogan, Y. L., Khairoutdinov, M. P., Lilly, D. K., Kogan, Z. N., and Liu, Q.: Modeling of stratocumulus cloud layers in a large eddy simulation model with explicit microphysics, J. Atmos. Sci., 52, 2923-2940, 1995.

Kogan, Y. L., Mechem, D. B., and Choi, K.: Effects of Sea-Salt Aerosols on Precipitation in Simulations of Shallow Cumulus, J. Atmos. Sci., 69, 463-483, 2012.

Larson, V. E. and Golaz, J.-C.: Using probability density functions to derive consistent closure relationships among higher-order moments, Mon. Weather Rev., 133, 1023-1042, 2005.
Larson, V. E., Wood, R., Field, P. R., Golaz, J.-C., Vonder Harr, T. H., and Cotton, W. R.: Systematic biases in the microphysics and thermodynamics of numerical models that ignore subgrid-scale variability, J. Atmos. Sci., 58, 1117-1128, 2001.

Larson, V. E., Schanen, D. P., Wang, M., Ovchinnikov, M., and Ghan, S.: PDF parameterization of boundary layer clouds in models with horizontal grid spacings from 2 to $16 \mathrm{~km}$, Mon. Weather Rev., 140, 285-306, 2012.

Nelson, K. J., Mechem, D. B., and Kogan, Y. L.: Evaluation of warm-rain microphysical parameterizations in mesoscale simulations of the cloudy marine boundary layer, Mon. Weather Rev., 144, 2137-2154, 2016.

Pincus, R. and Klein, S. A.: Unresolved spatial variability and microphysical process rates in large-scale models, J. Geophys. Res., 105, 27059-27065, 2000.

Rauber, R., Ochs III, H. T., Di Girolamo, L., Göke, S., and Snodgrass, E.: Rain in (shallow) cumulus over the ocean - the RICO campaign, B. Am. Meteorol. Soc., 88, 1912-1928, 2007.

vanZanten, M. C., Stevens, B. B., Nuijens, L., Siebesma, A. P., Ackerman, A., Burnet, F., Cheng, A., Couvreux, F., Jiang, H., Khairoutdinov, M., Kogan, Y., Lewellen, D. C., Mechem, D., Nakamura, K., Noda, A., Shipway, B. J., Slawinska, J., Wang, S., and Wyszogrodzki, A.: Controls on precipitation and cloudiness in simulations of trade-wind cumulus as observed during RICO, J. Adv. Model. Earth Syst., 3, M06001, doi:10.1029/2011MS000056, 2011. 